\title{
Ueber die Geschwindigkeit der Oxydationsprocesse im arteriellen Blutstrom.
}

Von

\section{w. Pfinger.}

Zwei französische Forscher, Estor und Saint-Pierre haben vor nunmehr drei Jahren die merkwürdige Behauptung als Resultat ihrer Untersuchungen aufgestellt, dass gemäss der Entfernung des Blutes vom Herzen der Sauerstoffgehalt sich in ausserordentlicher Weise verringere ${ }^{1}$ ).

Als Mittelzahl aus den Beobachtungen von Bernard und ihren eigenen finden Jene folgende Werthe:

1) für die Art. carotis . . . $16.16 \%$ Sauerstoff.

2) " " renalis . . $14.85 "$ "

3) " " " lienalis . . . $10.92 "$ "

4) " " cruralis . . . $5.79 "$ "

Wie gewöhnlich besagen diese Zahlen, wieviel Cc. eines bestimmGases, welches bei $0^{\circ} \mathrm{C}$. und dem Drucke einer Quecksilbersäule von 1 Meter Höhe gemessen wird, in $100 \mathrm{Cc}$. Blut enthalten seien.

Erwägt man, wie ausserordentlich schnell ${ }^{\circ}$ das Blut durch das Arteriensystem abfliesst, so würde eine ganz intensive Verbrennung in diesem vorhanden sein müssen, wenn sich eine so grosse Verschieden heit des Gasgehaltes für die Gefässe verschiedener Entfernung vom Herzen bewahrheiten sollte.

Da man bisher den Ort der Verbrennungsprocesse nicht in das Blut, sondern allgemein in die Gewebe verlegte, so ist es auffallend, dass die Theorie Estor's und Saint-Pierre's nicht lebhafteren Wider spruch gefunden, ja sogar sich Anhänger bei uns erwerben konnte.

1) Estor et Saint-Pierre. Du siège des combustions respiratoires. Journ. de l'anatomie et de la physiologie 1865. Mai p. 310 . 
W. Dybkowsky aus Kiew von den Behauptungen jener beiden französischen Forscher wie von ausgemachten Thatsachen ${ }^{1}$ ).

Ebenso schliesst sich F. Hoppe-Seyler selbst ausdrücklich Estor und Saint-Pierre an. Er beruft sich $^{2}$ ) auf einen zuerst von mir angestellten Versuch, welcher darthut, dass Blut, welches in einer Arterie zwischen zwei Unterbindungen zu stagniren gezwungen ist, bald dunkelvenös wird ${ }^{3}$ ). Derselbe Forscher beschreibt auch ${ }^{4}$ ) noch folgendes hierhergehöriges Experiment. "Von einem Hunde, welcher durch Verblutenlassen getödtet war, wurde sofort mit dem Tode die obere Hälfte der Aorta schnell isolirt und mit defibrinirtem Blute desselben Thieres in einem Probirglase bei $38^{\circ}$ digerirt. Nach 2 Stunden war der grösste Theil des Blutes in diesem Glase dunkelvenös, nur die Partieen des Blutes, welche von dem Arterienrohre weiter entfernt waren, hatten hellere Farbe behalten. Ein Stück des Arcus Aortae mit Carotis und Subclavia dextra waren in verdünnte Kochsalzlösung gebracht, einige Tropfen defibrinirten Blutes hinzugefügt und gleichfalls 2 Stunden bei $38^{\circ}$ digerirt, auch hierin war das Haemoglobin von Sauerstoff grösstentheils befreit."

Hoppe-Seyler schliesst aus diesen und anderen Versuchen ${ }^{5}$ ):

I. Das arterielle Blut verliert während seines Strömens durch die Arterien bereits einen Theil seines Sauerstoffs, den es in den Lungen aufgenommen hat.

II. Der Verlust dieses Sauerstoffs steht in keiner Beziehung zum Vorhandensein der fibrinbildenden Stoffe.

III. Derselbe ist aber abhängig von der Berührung des Blutes mit der lebenden Gefässwandung.

IV. Der Einfluss der Gefässwandung zeigt keine Fernwirkung ist also kein physikalischer, sondern ein chemischer.

V. Der Verlust des Sauerstoff's vom Oxyhaemoglobin des ar-

1) S. Dr. W. Dybkowsky. Einige Bestimmungen über die Quantität des mit dem Haemoglobin lose gebundenen Sauerstoffes in ,Medicinisch-chemische Untersuchungen von Felix Hoppe-Seyler. Heft I. Berlin 1866." pag. 123.

2) Hoppe-Seyler a. a. 0. pag. 136.

3) E. F. W. Pflüger. Ueber die Kohlensäure des Blutes. Bonn 1864. pag. 15.

4) F. Hoppe-Seyler. Medicinisch-chemische Untersuchungen. 1866. Heft I. pag. 137 .

5) Hoppe-Seyler a. a. O. p. 138. 
teriellen Blutes wird nicht durch Oxydation von Stoffen veranlasst, welche aus der Gefässwandnng in das Blut sich diffundiren, sondern der Sauerstoff wird an die Wandung selbst abgegeben; in dieser allein kann der Oxydationsvorgang zu suchen sein, welcher dem Blute bei seinem Strömen durch die Gefässe Sauerstoff entzieht.«

Hierauf sagt F. Hoppe-Seyler ${ }^{1}$ ) ausdrücklich:

"Das unter I angeführte Resultat ist eine Bestätigung der Angaben von Saint-Pierre und Estor."

Ich selbst habe den Letzteren ebenfalls wenigstens principiell eine weitere Stütze zugeführt, indem ich darthat, dass in dem lebendigen arteriellen Blute in der That Oxydationsprocesse continuirlich ablaufen, wonach obige Angaben Hoppe-Seyler's in geeigneter Weise $\mathrm{zu}$ modificiren sind.

Zuerst zeigte ich, dass lebendiges arterielles Blut, welches bei Körpertemperatur erhalten wird, nicht bloss in der lebendigen Arterie, sondern auch bei vollkommenem Luftabschluss in Gläsern mehr oder weniger schnell, oft ausserordentlich rasch dunkelt, $d . \mathrm{h}$. dem venösen Zustande zustrebt. Hierbei kommt es mit keinem Gewebe in Berührung und muss also selbst freien Sauerstoff in festere Verbindung überführen. Kühlt man frisch entleertes Blut der Arterie augenblicklich auf $0^{\circ} \mathrm{C}$. ab, so bleibt es sehr schön hellroth, weil die Kälte den Ablauf der Oxydationsprocesse verhindert oder doch ungemein verlangsamt ${ }^{2}$ ).

Ich habe aber ferner bewiesen, dass wenn man mit Hülfe der Quecksilberpumpe arteriellem Blute den Sauerstoff entzieht, um so mehr von diesem Gase gewonnen wird, je geschwinder die Auspumpung geschieht, weil dem Sauerstoff keine Zeit bleibt, in feste Verbindungen überzutreten. Mit Hülfe der momentanen von mir entdeckten Entgasungsmethode erhielt ich aus denselben Blutarten als Mittel für das arterielle Blut $16.9 \%(0 \circ \mathrm{C}$. und 1 Meter $)$ oder $22.2 \%$ $\left(0^{\circ} \mathrm{C}\right.$ und 0.76 Meter), während mit Hülfe der bisher gebräuchlichen Methode nur $15.3 \%\left(0^{\circ} \mathrm{C}\right.$ und 1 Meter $)$ oder $20.1 \%$ gewonnen werden konnten ${ }^{3}$. Sehr bemerkenswerth für diese Resultate bleibt aber

1) Hoppe-Seyler a. a. O. pag. 138.

2) E. Pflüger. Centralblatt f. d. medicinischen Wissenschaften. 1867. Nro. 21.

3) E. Pflüger. Die normalen Gasmengen des arteriellen Blutes nach verbesserten Methoden. Centralblatt für die medicinischen Wissenschaften. 1867. Nro. 46. 
dass, was ich bisher nicht hinreichend anderwärts betont habe, nicht viel darauf ankommt, ob die eine Portion desselben Blutes in einer bestimmten Pumpe 1 Stunde länger zur vollkommenen Entgasung beansprucht als die zweite Portion desselben in einer anderen Pumpe. Die Resultate welche beide Proben geben, sind nahezu identisch, wenn diese letzteren es auch waren.

Worauf Alles ankommt, ist, dass die Partie lebendigen Blutes, welche mehr Sauerstoff liefern soll, wirklich fast augenblicklich $d$. h. in circa $1-2$ Minuten entgast wird. Hieraus ergiebt sich, dass der vorwiegend starke Sauerstoffiverbrauch nur im lebendigen Blute stattfindet und dann schnell abschliesst, um sehr gering zu werden, wenn die Gerinnung einmal abgelaufen ist. Ganz in Uebereinstimmung hiermit ist die von N. Zuntz gefundene wichtige Thatsache, dass die stärkste Abnahme der Alkalescenz des frisch entleerten arteriellen Blutes nur in den ersten Minuten nach dem Verlassen des lebendigen Körpers beobachtet wird ${ }^{1}$ ).

Wenn man an frisch entleertem BJute das schnelle Dunkeln und den hiermit einhergehenden Sauerstoffverbraitch in das Auge fasst, und dann die langdauernde Unveränderlichkeit der Farbe und die geringe Variation des Gasgehaltes von der Luft abgeschlossenen arteriellen Blutes, welches einer Temperatur von $38^{\circ} \mathrm{C}$. ausgesetzt wird, so tritt die Frage auf, wie diese Differenz des Verhaltens zu verstehen sei.

Man muss zunächst daran denken, dass mit dem Gerinnungsprocess das Leben des Blutes abschliesst; denn es wird ja hiermit, worauf die Untersuchungen von N. Zuntz ${ }^{2}$ ) hinweisen, offenbar todenstarr. Die wesentlichsten Substanzen, welche das lebende Ḅlut zusammensetzen, bewahren indessen allerdings auch im defibrinirten ihre chemischen Eigenschaften, da solches Blut in den Körper eines verbluteten scheintodten Thieres durch Transfusion übergeführt, das Bewustsein zurïckruft und die normalen Lebensprocesse wieder zu unterhalten fähig ist. Die Frage, warum das lebendige Blut in einen anderen Zustand übergeht, wenn es den lebendigen Körper verlässt,

1) N. Zuntz. Centralblatt für die medicinischen Wissenschaften. 1867. Nro. 51.

2) N. Zuntz. Beiträge zur Physiologie des Blutes. Inaugural-Dissertation. Bonn 1868. pag. 20 u. flgde. - Ferner N. Zuntz. Centralblatt für die medicinischen Wissenschaften. 1867. Nro. 51. 
ist wohl identisch mit der, warum es gerinnt, d. h., warum es todenstarr wird oder abstirbt. Das Blut gerinnt dann, wenn es irgend einen Körper berührt, der nicht normale Gefässwand oder normales Blut ist. Die Gerinnung ist eine in das Gebiet der Contactwirkungen gehörige Erscheinung.

Jede andere Moleculanrordnung als die in der innersten Schicht der normalen Gefässwand vorhandene stört den Gleichgewichtszustand gewisser Atomencomplexe der Eiweissstoffe des Blutes, so dass eine Reihe von Metamorphosen eintritt, deren in die Augen fallendste die Ausscheidung von Faserstoff ist. Es bleibt dahingestellt, ob diesem Processe die Fibringeneratoren erst ihre Entstehung verdanken, oder ob einer von ihnen oder beide in der That im Blute praefornirt sind.

Hieraus ergiebt sich also, dass die Vorsicht erheischt, die scheinbare Indifferenz des defibrinirten Blutes gegen Sauerstoff nicht als Leitstern zu wählen, wo es sich um das lebendige Blut handelt, für welches eben die Zustände und Eigenschaften jenes nicht ganz maassgebend sind.

Ich habe nun das rasche Dunkeln auch an der rings isolirten sonst ganz unversehrten und in ihren normalen Verbindungen befindlichen Art femoralis des Hundes gesehen, wenn ich das Blut durch Compression des Gefüsses mit einer Klemmpincette für einige Augenblicke zum Stagniren zwang. Dieser Versuch setzt natürlich eine dünne Gefässwand voraus.

Alexander Schmidt ${ }^{1}$ ) hat endlich in Ludwig's Laboratorium den Nachweis geliefert, dass im Erstickungsblute leicht oxydirbare Stoffe in grösserer Menge vorkommen. Wenn man solchem Blute, welches bekanntlich nur Spuren von Sauerstoff enthält, künstlich dieses Gas zuführt und digerirt, so verschwindet der Sauerstoff, wie Schmidt und ich gezeigt haben, sehr schnell, während Kohlensäure allmählich neugebildet wird. Arterielles Blut zeigt diese Erscheinung in geringerem Maasse, so dass demnach im Venenblute stets Stoffe in grösserer oder geringer Menge sein werden, welche Sauer-

1) Alexander Schmidt. Die Athmung innerhalb des Blutes. Zweite Abhandlung. - Aus dem physiologischen Institute zu Leipzig. Vorgelegt von dem wirkl. Mitgliede C. Ludwig im Berichte der Königl. sächs. Gesellschaft der Wissenschaften. Mathematisch-physikalische Classe. Sitzung am 9. November 1867. 
stoff consummiren und wohl zum Theil auch noch nach dem Arteriensystem übertreten. Denn es ist doch kaum zu bezweifeln, dass was für Erstickungsblut festgestellt ist, welches wir für höchstgradig venös ansehen müssen, wenn auch in geringerem Maasse für das gewöhnliche Venenblut Geltung habe.

Dass also innerhalb der arteriellen Blutgefässe ein continuirlicher Sauerstoffverbrauch stattfindet, das wird zu bestreiten heutigen Tages Niemand mehr versuchen.

Darum aber ist die von Estor und Saint-Pierre aufgestellte merkwürdige Behauptung noch bei Weitem nicht gerechtfertigt. Denn ihr Schwerpunkt liegt in der enormen Intensität des Verbrennungsprocesses, der im Arterienrohre ablaufen soll und als so bedeutend dargestellt wird, dass alle thierische Oxydation dann in das Arteriensystem verlegt werden müsste.

Ich will nun zunächst den Nachweiss liefern, dass die quantitativen Angaben von Estor und Saint-Pierre mit einer Reihe wohl begründeter Thatsachen im Widerspruche stehen. Wenn die Behauptungen jener Forscher richtig wären, dann würde das Blut auf seinem Wege vom Herzen bis zur Arteria cruralis etwa $2 / 3$ seines Sauerstoffgehaltes verlieren; denn die A. Carotis soll im Mittel 16.27, die A. cruralis $5.8 \%$ Sauerstoff enthalten. Es büsste das Blut also auf diesem Wege $10.4 \%$ Sauerstoff ein. Nach den Untersuchungen von Schöffer ${ }^{1}$ ) und Ludwig $^{2}$ ), die volles Vertrauen verdienen enthält:

\begin{tabular}{|c|c|c|c|c|c|}
\hline & & N. & 0. & $\mathrm{Co}_{2}$ & $\begin{array}{l}\text { Unterschied des } \\
\text { Sauerstoffs. }\end{array}$ \\
\hline Mitt & Arterienbl & 2.04 & 14.61 & $29.99\}$ & מ. \\
\hline Mittleres & Venenblut & 1.32 & 9.05 & $34.40\}$ & \\
\hline
\end{tabular}

Diese Untersuchungen sind mit dem Herzblut angestellt, so dass man das Resultat der Veränderung vor sich hat, welche das Blut während des grossen und kleinen Kreislaufs erfährt.

Nach den Angaben von Estor und Saint-Pierre würde das Blut also auf einem Theile seiner Bahn 10.4\% Sauerstoff verlieren, während wir doch nach den so eben citirten sicheren in Ludwig's La-

1) Schöffer. Sitzungs-Berichte der K. K. Academie der Wissenschaften zu Wien 41 Bd. pag. 589.

2) C. Ludwig. Zusammenstellung der Untersuchungen über Blutgase, welche aus der physiologischen Anstalt der Josephsacademie hervorgegangen sind. (Separatabdruck aus den medicinischen Jahrbüchern. Zeitschrift der K. K. Gesellschaft d. Aerzte in Wien. pag. 16 u. 6). 
horatorium festgestellten Thatsachen wissen, dass es während des ganzen Kreislaufs nur 5.6\% einbüsst, also nicht $2 / 3$, sondern etwa $1 / 8$ seines Totalgehaltes. Wollte man sich darauf berufen, dass bei Estor und Saint-Pierre die Mittelwerthe für das Blut der A. cruralis zufällig abnorm niedrig, bei den von Schöffer angestellten Versuchen zufällig die Differenz zwischen Arterien- und Venenblut zu zu klein sei, so würde noch die Betrachtung der zeitlichen Verhältnisse die von den genannten Forschern behauptete rapide Verminderung des Sauerstoffs im Arterienrohre ad absurdum führen. Nach den genauen Untersuchungen Volkmanns beträgt die mit dem Hämodromometer gemessene Strömungsgeschwindigkeit des Blutes in der Aorta $400 \mathrm{Mm}$. in der Secunde. Rechnet man den Abstand der Carotis von der Femoralis beim Menschen zu circa $500 \mathrm{Mm}$. so würde das Blut inmaximo 2 Secunden brauchen, um aus dem An fange der Aorta nach der Cruralis zu gelangen. Nach Vierordt dauert ein Kreislauf beim Hunde 15.2, beim Menschen 21.5 Secunden. Wenn nun das Blut in dieser Zeit von 15 bis 21 Secunden 5,6\% Sauerstoff verliert, d. h. consummirt, so würde es, selbst wenn man alle Oxydationsprocesse ins Blut verlegte, was doch gewiss nicht richtig ist, in 2 Secunden nur $0,7 \%$ oder $0,5 \%$ Sauerstoff einbüssen können. Halten wir fest, dass diess nur dann gilt, wenn der Sauerstoffverbrauch ganz constant ist, d. h. wenn ein Cubiccentimeter Blut in jedem Zeittheilchen dieselbe Sauerstoffmenge verliert. In Wirklichkeit geschieht aber der Sauerstoffverlust am schnellsten in den Capillaren, in den Arterien am langsamsten und in den Venen wahrscheinlich etwas schneller als in den Arterien. Demgemäss ist obiger Werth von $0,5-0,7 \%$ noch viel zu gross. Dass der Sauerstoffverbrauch in den Capillaren bei weitem stärker ist, als in den Arterien ergiebt sich nicht daraus, dass Arterienblut roth und Venenblut dunkel erscheint, wie man gewöhnlich angiebt, sondern daraus, dass in einem abgeschlossenen Gefäss von Glas über Quecksilber aufgefangenes lebendiges Arterienblut oder solches durch Arteriencompression zur Stagnation in seinem natürlichen Behälter gezwungenes niemals in soviel Zeit als der Capillarkreislauf etwa datert so stark dunkelt, wie in den Capillaren. Da ferner nach den Untersuchungen Alexander Schmidt's über Erstickungsblut nothwendig das Venenblut mehr leicht oxydirbare Stoffe als das Arterienblut enthält, so muss die Energie der Oxydation in der Vene stärker als in der Arterie sein. Da diese Stoffe durch die Capillaren in das Blut über- 
treten, wo sie noch reichlichen Sauerstoff finden, so wird das Maximum der Verbrennung in dem Blute selbst abermals in das Capillarrohr verlegt werden müssen. Das Dunkeln abgeschlossenen lebendigen Arterienblutes gestaltet sich so, dass es in den ersten Secunden sehr schnell abläuft, dann äusserst langsam vorschreitet. Die wahrscheinlichste Erklärung scheint mir darin begründet zu sein, dass gewöhnlich aus dem Venensystem die noch nicht ganz verbrannten leicht oxydirbaren Stoffe nach dem Arteriensystem gelangen, um hier schnell ganz oxydirt zu werden. Sobald dies geschehen ist, schreitet die langsame Selbstoxydation des Blutes allein weiter vor, wie auch eine geringe Abgabe von Sauerstoff an die Gefässwand statt haben mag. Wie gross nun thatsächlich der Sauerstoffverlust in der Zeit des Capillarkreislaufs ausfällt, ist allerdings nicht direct festgestellt; bedenkt man aber, wie bedeutend die Farbendifferenz der Arterie und irgend einer aus einem Organe kommenden Vene stets gefunden wird, und dass die Gasanalysen ebenso nicht merkbar kleinere Unterschiede ergeben, als zwischen dem Blut des rechten und linken Herzens, so wird man wohl ohne Bedenken annehmen dürfen, dass innerhalb der Zeit des Capillarkreislaufs, der etwa 1 Secunde dauert, das Blut den bei weitem grössten Theil der Sauerstoffmenge einbüsst, die es während eines ganzen Kreislaufs überhaupt überall und fortwährend verausgabt. Daraus ergiebt sich dann ferner, dass es während der kurzen Zeit seines Verweilens in den Arterien eben nur Spuren verlieren kann, die mit 0,1 bis $0,2 \mathrm{Vol} . \%$ wohl noch $\mathrm{zu}$ hoch gegriffen sein dürften. Diess sind aber Werthe, welche mit den feinsten Methoden und bei sorgfältigster Arbeit nur durch sehr lange Versuchsreihen festgestellt werden könnten. Denn diese Werthe sind von einerlei Ordnung mit den Beobachtungsfehlern.

Somit könnten Estor und Saint-Pierre nur noch die Ausflucht bleiben, dass sie behaupteten, es seien ihre Werthe als Maass der Intensität der Verbrennung im Arteriensysteme wohl etwas zu hoch. Denkbar bleibe es aber, dass ein allerdings noch unbekanntes und räthselhaftes Moment in der Arterie plötzlich die Oxydation zu grösserer Stärke anrege, wie das ja auch in den Capillaren der Fall sei.

Um auch diesem Einwande noch Rechnung zu tragen, habe ich eine Reihe von Experimenten angestellt, um die Angelegenheit vollkommen zum endgültigen Abschlusse zu bringen. Es handelt sich 
jetzt also zunächst um die Vergleichung des Blutes der A. carotis und femoralis auf einen etwaigen Unterschied des Gasgehaltes. Hierzu darf man natürlich nur das Blut desselben Thieres benutzen, da das von verschiedenen $\mathrm{zu}$ ausserordentlich verschiedene Zusammensetzung und Gasgehalt selbst bei derselben Species darbietet, was ja genugsam bekannt ist. Da aber, wie ich gezeigt habe, der Gasgehalt bei demselben Individuum periodisch auf- und abschwankt, so muss in demselben Moment der Aderlass an beiden Arterien beginnen und in demselben Moment aufhören. Es ist aber ferner nothwendig, dass die relativen Strömungsgeschwindigkeiten während des Blutlassens aus beiden Gefässen absolut constant bleiben. Ich nahm deshalb zwei gleich hohe, gleich weite, identisch gestellte, mit Quecksilber gefüllte und gleich tief in Quecksilber tauchende Cylinder, legte in A. carotis und A. femoralis zwei identische Canülen, die mit identischen Gummischläuchen verbunden waren, und verband das andere Ende dieser mit identischen Glasröhrchen, welche aufgebogen waren, damit man aus ihnen das Blut bequem in die $\mathrm{Cy}$ linder einlassen konnte. Sobald das vordringende Blut die Luft aus den Schläuchen ausgetrieben und einige Zeit aus den Glasröhrchen gespritzt hat, comprimirt man die Gummischläuche mit den Fingern beider Hände. Die Gehülfen führen dann die Glasröhrchen unter die Quecksilbercylinder. In ein und demselben Moment lässt man dann die Compression mit den Fingern aufhören, sodass das Blut in den Cylindern aufzusteigen beginnt. Es ist nothwendig, dass e ine Person diese Compression mache und plätalich aufhebe. Wenn der Versuch gelingt, senkt sich das Quecksilber gleich schnell in beiden Cylindern, sodass nahezu gleichviel Blut aus beiden Arterien erhalten wird. Hat man genug, so comprimirt man beide Blutzuleitungsschläuche wieder mit den Fingern beider Hände in demselben Moment. Diess gelingt recht gut bei strengster Aufmerksamkeit. Ist das Blut nicht gleichschnell in beide Cylinder ergossen worden, so muss der Versuch verworfen werden, was mir leider öfter geschah. Die Quantität des Blutes soll, besonders wenn es schnell fliesst, nicht zu klein genommen werden, weil die in der ersten und letzten Secunde fliessende Menge einen Fehler bedingt, der möglichst verklejnert werden muss. Denn, wie man weiss, schwankt mit der Athmung der Gasgehalt periodisch, sodass am Anfange des Aortensystems nicht derselbe Gasgehalt wie am Ende gefunden zu werden braucht. Im ersten Moment des Aderlasses kann man deshalb aus der A. 
femoralis Blut erhalten, welches von einer andern Systole herrührt als dasjenige, welches eben im Anfange des Aortensystemes sich vorfindet und aus der Carotis abströmt. Diese Anfangsmengen können deshalb different sein. Dasselbe gilt aus ähnlichem Grunde für die Endmengen.

Das wichtigste Moment zur Entscheidung unserer Frage liegt nun offenbar in der Vergleichung der Farbe des auf diese Weise gewonnenen Blutes der A. carotis und A. femoralis. Da Venenund Arterienblut die Farbendifferenz dem Unterschiede des Sauerstoffgehaltes verdankt, und dieser letztere trotz der Grösse jener doch nicht mehr als $5.6 \%$ im Mittel nach den oben angeführten Untersuchungen von Ludwig und Schöffer beträgt, so müssten so colossale Unterschiede des Gasgehaltes, wie sie nach Estor und Saint-Pierre für Carotis- und Femoralisblut vorhanden sind, durch einen evidenten Farbenunterschied sich deutlich bemerkbar machen. Diese Beobachtungen sind darum so ausserordentlich wichtig, weil man die beiden Blutarten direct neben einander unmittelbar nach dem Verlassen des Körpers vor sich hat, ehe sie Zeit behalten sich weiter zu verändern. Diese Methode ist frei von allen Bedenken, die den Resultaten etwa noch anhaften können, welche durch Auspumpung mit der Quecksilherpumpe erhalten sind.

Ich stellte die beiden das Blut der A. carotis und A. femoralis aufnehmenden Cylinder in helles licht identisch gegen dasselbe geneigt und betrachtete vergleichend sofort beim Ausfliessen mit anderen Zeugen (Herr Dr. E. Kemmerich und Herr Dr. N. Zuntz) die Farbe. Wir waren aber niemals im Stande den geringsten Fa:benunterschied $\mathrm{zu}$ entdecken, wiewohl ich den Versuch häufig wiederholt habe. Herr Dr. W. Preyer, der in colorimetrischen Untersuchungen eine grosse Uebung besitzt, überzeugte sich ebenfalls von der vollkommen gleichen Röthe beider Blutarten. Trotz der grossen Einfachheit dieses Versuches würde ich ihn doch für genügend zur Widerlegung der Behauptungen Estor's und Saint-Pierre's halten.

Um aber auch den letzten Zweifel auszuschliessen habe ich noch einige gasometrische Differentialanalysen angestellt, nachdem das über Quecksilber aufgefangene Blut in bekannter Weise bei niederer Temperatur defibrinirt worden war. Solche Differentialversuche sind allerdings bereits von Hirschmann ${ }^{1}$ ) angestellt worden,

1) Heinrich Hirschmann. Ein Beitrag zur Frage über den Ort der 
der darthat, dass so grosse Differenzen, wie sie nach Estor und Saint-Pierre vorhanden sein sollen, gewiss nicht existiren. In dieser Untersuchung, welche unter der Leitung des Prof. Sczelkow in Charkow ausgeführt wurde, ergaben sich folgende Resultate:

$\mathrm{T}$ a b e $11 \mathrm{e}$,

die den Gasgehalt in 100 Volumina Blut angiebt:

\begin{tabular}{l|l|r|r|r|c}
\hline & & $\mathrm{CO}_{2}$ & 0. & $\mathrm{~N}$. & $\begin{array}{c}\text { Gesammt- } \\
\text { menge. }\end{array}$ \\
\hline Vers. I. & & & & & \\
\hline Vers. II. & A. Carotis & 18.432 & 20.800 & 2.756 & 41.988 \\
A. Femoralis & 14.777 & 19.563 & 4.531 & 38.871 \\
Vers. III. & A. Carotis & 30.918 & 12.861 & 1.974 & 45.753 \\
A. Femoralis & 32.492 & 12.857 & 1.892 & 47.241 \\
Vers. IV. & A. Carotis & 28.228 & 10.283 & 1.710 & 40.221 \\
A. Femoralis & 27.927 & 11.547 & 1.557 & 41.031 \\
Vers. V. & A. Carotis & 29.158 & 12.325 & 2.455 & 43.938 \\
A. Lienalis & 27.283 & 13.882 & 2.401 & 43.566 \\
& A. Carotis & 17.702 & 8.668 & 0.934 & 27.304 \\
A. Lienalis & 16.874 & 7.878 & 0.823 & 25.575
\end{tabular}

Die Volumina beziehen sich auf $0^{\circ} \mathrm{C}$. und den Druck einer Quecksilbersäule von $1 \mathrm{M}$. Höhe.

Die Differenzen, welche Sczelkow und Hirschmann auffanden, überstiegen für den procentigen Gehalt an Sauerstoff nie $1.5 \%$ und fanden auch nicht immer in gleichem Sinne statt. So fanden jene Forscher in Versuch $I$, ndass das Blut der A. femoralis ärmer an Sauerstoff sei als das der A. Carotis; im Versuch III war jedoch das Gegentheil der Fall «. Versuch $I$ ist freilich wegen Lufteintritt offenbar fehlerhaft. Ebenso zeigte sich bei Vers. IV, dass das Blut der A. Lienalis mehr Sauerstoff als das der Carotis darbot. Da der Beobachtungsfehler von Sczelkow für den Sauerstoff $1.5 \%$ beträgt, so lassen sich Veränderungen des Blutes, welche unterhalb dieses Werthes bleiben, durch eine so kleine Zahl von Versuchen nicht mehr darthun. Es wäre aber ein bereits ungeheuer wichtiges

Kohlensäurobildung im Organismus. Archiv für Anatomie, Physiologie und wissenschaftliche Medicin, herausgegeben von Reichert u. du Bois-Reymond. Leipzig 1866, p. 502-517. 
Factum, wenn das Blut von dem Anfange der Carotis bis zur Femoralis nur $1 / 2$ Procent Sauerstoff verlöre, weil eben die Zeit so kurz ist. Jener grosse Beobachtungsfehler, der in den Analysen von Sczelkow und H. Hirschmann zu Tage tritt, ist dadurch bedingt, dass diese Forscher, wie sie selbst angeben, nnicht die Möglichkeit hatten, das Blut aus beiden Arterien gleichzeitig zu sammeln; deshalb verfloss zwischen dem Blutaufsammeln aus der ersten und zweiten immer ein gewisser Zeitraum ". Sczelkow und Hirschmann sagen: dass sie aus diesem Grunde schon a priori nicht erwarten durften, dass der Sauerstoffgehalt in beiden Blutportionen absolut gleich bleibe. "Die Richtigkeit dieser Erklärung lässt sich darlurch beweisen, dass der grössere Sauerstoffgehalt, wie aus der oben angeführten Tabelle ersichtlich, bald im Blute der vom Herzen weiter, bald in dem der vom Herzen weniger entfernten Arterie vorkommt«. Ich muss mich dieser Ansicht der genannten Physiologen durchaus anschliessen, da man ja hier, weil keine Doppelanalysen vorliegen, an Beobachtungsfehler denken könnte, welche die Ursache der Differenzen wären. Ich habe solche gedoppelte Differentialversuche angestellt und in der That mich überzeugt, dass der Gasgehalt im Blute im fortwährenden sehr schnellen Auf- und Abschwanken begriffen ist, worauf ich bereits an einer anderen Stelle hinwies ${ }^{1}$ ).

Weil es aber darauf ankam zu constatiren, ob nicht dennoch kleinere Differenzen zwischen dem Blut der Carotis und Femoralis vorkommen möchten, wollte ich gleichzeitig beide Gefässe anzapfen, um den durch die zeitlichen Schwankungen des Gasgehaltes bedingten Fehler auf ein Minimum herabzudrücken.

Ich gehe demgemäss nunmehr zur Beschreibung meiner eigenen Versuche über.

\section{Versuch I.}

Grosser Hund. Das Blut floss aus einem engen Glasröhrchen gleichmässig und langsam. Es wurden beide Portionen mit zwei fast congruenten Pumpen gleichzeitig ausgepumpt. Specifisches Gewicht $=1,046$. Der Hund hat vor 3 Stunden bereits einen Aderlass erlitten. Das zuerst aufgefangene Blut ergab ein specifisches Gewicht von 1,049 .

1) E. Pflüger, Ueber die Ursache der Athembewegungen, sowie der Dyspnoë und Apnoë in diesem Archiv. Bd. I. p. 70 u. f. 


\section{Res u lt at:}
A. Carotis.
A. Femoralis.

$11.1 \%$ Sauerstoff

$11.2 \%$ Sauerstoff

$26.6 \%$ Kohlensäure

$26.8 \%$ Kohlensäure

$1.3 \%$ Stickstoff

$1.3 \%$ Stickstoff.

Die hier auftretenden Unterschiede sind so klein, dass sie von Beobachtungsfehlern herrühren können oder von nicht ganz gleichmässigem Fliessen und entsprechen in Nichts den Angaben von Estor und Saint-Pierre. Denn die Femoralis enthält zwar $0,2 \%$ Kohlensäure mehr als das Carotisblut, aber ebenso auch $0,1 \%$ Sauerstoff mehr.

\section{Versuch II.}

Aeusserst kräftige Dogge, die einem Schlächter angehört, also wohl hauptsächlich Fleischnahrung erhalten hatte. Das specifische Gewicht des Blutes erweist sich ungemein hoch $=1.066$.

\section{Resultat:}
A. Carotis.
A. Femoralis.

$18.8 \%$ Sauerstoff $18.7 \%$ Sauerstoff $26.7 \%$ Kohlensäure $26.8 \%$ Kohlensäure $1.1 \%$ Stickstoff $1.2 \%$ Stickstoff.

Bei diesem Versuche tritt nun in der That ein Unterschied hervor, der zwar sehr klein ist, aber in der Richtung der Angaben von Estor und Saint-Pierre liegt. Denn das Blut der A. femoralis enthält $0.1 \%$ Sauerstoff weniger und $0.1 \%$ Kohlensäure mehr. Die Grösse dieses Werthes ist von vorneherein nicht als undenkbar zu bezeichnen. Um aber darauf ein Gewicht legen zu kömmen, müssten sehr grosse Reihen von Versuchen angestellt werden, die mit sehr grossen Schwierigkeiten verknüpft sind, weil die relativen Ausflussgeschwindigkeiten für das Blut der Carotis und Femoralis für längere Zeit constant erhalten werden müssen. Vielleicht würde man am ehesten noch mit einem communicirenden Röhrensysteme zum Ziele gelangen, wie es von Ludwig und Alexander Schmidt in sinnreicher Weise angewandt worden ist ${ }^{1}$ ). Wenn es in einer längeren natürlich mit der äussersten Genauigkeit durchgeführten Ver-

1) Alexander Schmidt, Ueber die Kohlensäure in den Blutkörperchen. Erste Abhandlung. Aus dem physiologischen Institute zu Leipzig. Vorgelegt vom wirklichen Mitgliede Prof. C. Ludwig im Berichte der Kön. Sächs. Gesellschaft der Wissenschaften. Mathem.-physikal. Classe. Sitzung am 21. Mai 1865. 
suchsreihe festzustellen gelänge, dass dieser kleine Unterschied constant wäre, so würde das doch ein sehr wichtiges Factum sein, wegen der ausserordentlichen Kürze der Zeit, innerhalb welcher er eingetreten ist Denn wenn ein Bluttheil in 12 Secunden $0.1 \%$ Sauerstoff theils für sich, theils für die Gefässwand braucht, so würde dies für einen ganzen Kreislauf $1 \%$ Sauerstoff ausmachen, $d . h$. nahe $20 \%$ der gesammten während dieses Zeitraumes consumirten Menge. Dem Blute müsste damn ein ganz intensiver Stoffwechsel resp. Oxydationsprocess zugestanden werden.

Es schien mir nun endlich noch ein anderer Weg übrig zu bleiben, die in Rede stehende Frage zu entscheiden, nämlich der der Mittelwerthe oder derselbe, den Estor und Saint-Pierre einschlugen.

Fs sind bis jetzt eine grosse Zahl von Gasanalysen gemacht, die sich auf das arterielle Blut des Hundes beziehen. Wollte man Mittelwerthe derselben für Carotis und Femoralis verwenden, so müssten alle Experimente nach nahezu derselben Methode ausgeführt sein; also die Entgasung mit Hülfe der Quecksilberpumpe. Keine Analyse dürfte verwerthet werden, die von einem Hunde stammt, der schon einmal zu irgend welchen physiologischen Experimenten benutzt worden ist. Merkwürdigerweise sind alle hier zu verwendenden Analysen wie ich sehe mit Carotisblut angestellt und zum Glück besitze ich selbst nun ausser zahlreichen mit denselben ausgeführten auch sehr viele vom Blute der A. femoralis, an welche sich einige $H$. Hirschmann anreihen. Die meisten der Versuche, welche ich hier. mittheile, sind noch nicht publicirt. Damit meine Werthe mit denen von Ludwig und seinen Schülern vergleichbar seien, lasse ich die bei momentaner Entgasung enthaltenen Werthe fort. Ich gebe demgemäss die folgende Tabelle. 
Tabelle.

Gasgehalt des arteriellen Blutes in der Arteria carotis, bezogen auf den Druck von 1 Meter und $0^{\circ} \mathrm{C}$.

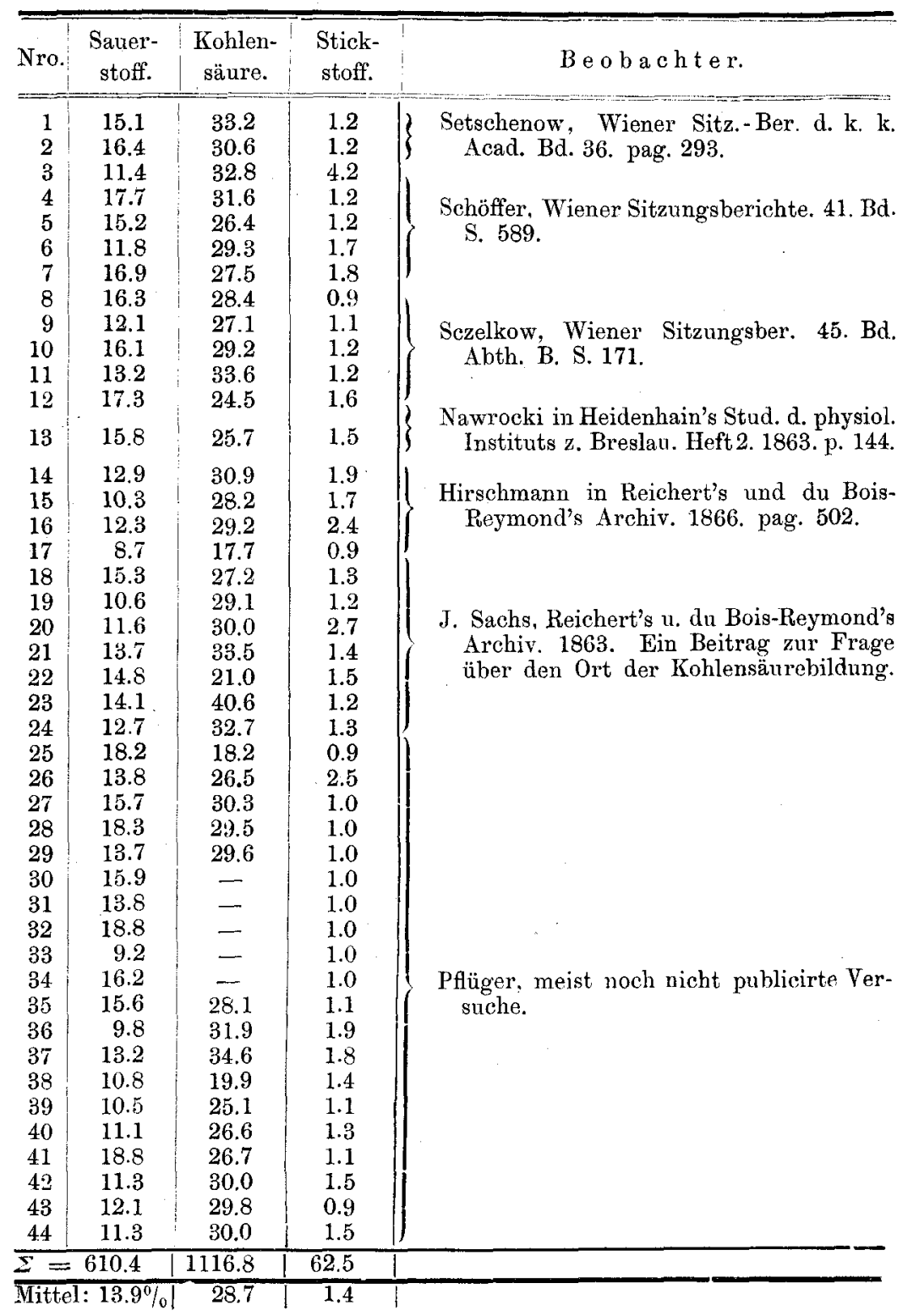


Ueber die Geschwindigkeit d. Oxydationsprocesse im arteriellen Blutstrom. 289

$$
\text { T a belle. }
$$

Gasgehalt des arteriellen Blutes in der Arteria femoralis, bezogen auf den Druck von 1 Meter und $0^{\circ} \mathrm{C}$.

\begin{tabular}{|c|c|c|c|c|}
\hline Nro. & $\begin{array}{l}\text { Sauer- } \\
\text { stoff. }\end{array}$ & $\begin{array}{l}\text { Kohlen- } \\
\text { säure. }\end{array}$ & $\begin{array}{l}\text { Stick- } \\
\text { stoff. }\end{array}$ & B e o b a chter. \\
\hline 1 & 12.9 & 32.5 & 1.9 & \multirow{27}{*}{$\begin{array}{l}\text { Pflüger, mit wenigen Ausnahmen noch } \\
\text { nicht publicirte Versuche. }\end{array}$} \\
\hline 2 & 11.5 & 27.9 & 1.5 & \\
\hline 3 & 10.3 & 31.8 & 1.1 & \\
\hline 4 & 12.3 & 39.8 & 3.6 & \\
\hline 5 & 14.6 & 23.4 & 1.1 & \\
\hline 6 & 16.0 & 32.3 & 1.2 & \\
\hline 7 & 16.6 & 32.4 & 1.0 & \\
\hline 8 & 15.0 & 31.7 & 1.0 & \\
\hline 9 & 16.0 & 30.0 & 2.5 & \\
\hline 10 & 15.2 & 28.1 & 1.3 & \\
\hline 11 & 15.7 & 39.0 & 1.4 & \\
\hline 12 & 14.2 & 27.2 & 1.6 & \\
\hline 13 & 13.6 & $\ldots$ & 1.0 & \\
\hline 14 & 14.3 & 34.6 & 1.8 & \\
\hline 15 & 10.5 & 18.4 & 1.6 & \\
\hline 16 & 11.6 & 24.6 & 1.1 & \\
\hline 17 & 11.2 & 26.8 & 1.3 & \\
\hline 18 & 18.7 & 26.8 & 1.2 & \\
\hline 19 & 11.7 & 29.8 & 1.4 & \\
\hline 20 & 11.0 & 29.6 & 2.2 & \\
\hline 21 & 11.7 & 29.8 & 1.4 & \\
\hline 22 & 14,4 & 36.9 & 1.4 & \\
\hline 23 & 18.6 & 24.8 & 1.2 & \\
\hline 24 & 14,4 & 29.8 & 1.2 & \\
\hline 25 & 16.2 & 27.5 & 1.7 & \\
\hline 26 & 14.8 & 30.9 & 1.8 & \\
\hline 27 & 15.5 & 23.0 & 3.2 & \\
\hline \multicolumn{2}{|c|}{$\Sigma=378.5$} & 769.4 & 42.7 & \\
\hline \multicolumn{2}{|c|}{ Mittel: $13.99 \%$} & $29.5 \%$ & $\overline{1.5}$ & \\
\hline
\end{tabular}

Wie man also sieht, ergiebt sich für die Mittelwerthe:

\begin{tabular}{c|c|c|c}
\hline Blutart & Sauerstoff. & Kohlensäure. & Stickstoff. \\
\hline Carotis & $13.9 \%$ & $28.7 \%$ & $1.4 \%$ \\
\hline Femoralis & $13.99 \%$ & $29.5 \%$ & $\mathbf{1 . 5 \%} \%$ \\
\hline
\end{tabular}

Für den Sauerstoff und Stickstoff fallen die Mittelwerthe identisch aus; die Femoralis giebt noch $0.1 \%$ Sauerstoff und Stickstoff mehr. Für die Kohlensäure liess sich, da ihre Werthe so ungeheuer schwanken, erst aus einer noch grösseren Zahl von Versuchen eine vollkommene Congruenz der Werthe erwarten. Doch beträgt die Abweichung für die Femoralis auch nur $+0.8 \%$. Obige Tabelle, die sich auf eine so grosse Zahl von Versuchen zuverlässiger Forscher stützt, beansprucht einen hohen Werth. Die Mittelzahl für 
den Sauerstoff ist unerwartet klein. Das hat wohl zwei Gründe. Ich habe bewiesen 1), dass bei der Art des Auspumpens, wie es bei obigen Versuchen innegehalten ist, im Mittel $1.6 \%$ Sauerstoff verloren gehen, welche zu obiger Mittelzahl addirt $14.5 \%$ ergeben. Dieser Werth liegt unter dem von mir aufgestellten von $16.9 \%$ noch um $1.4 \%$. Es ist unzweifelhaft und jedem mit solchen Untersuchungen Vertrauten bekannt, dass recht lebenskräftige Thiere einen höheren Sauerstoffgehalt in ihrem Blute beherbergen, als schwächliche. Als ich beabsichtigte, den normalen Gehalt an Gasen im normalen arterjellen Blute nochmals mit verbesserten Methoden festzustellen, nahm ich desshalb keine schwächlichen, sondern gesunde, kräftige, lebensfrische Hunde. In unserer obigen Tabelle aber sind alle möglichen Versuche von den verschiedensten Beobachtern, die nicht immer ein besonderes Interesse daran haben konnten, an prächtigen Thieren zu arbeiten. Deshalb sind obige Werthe gleichsam die Mittelwerthe für die oft nicht in dem besten Zustande befindlichen Hunde, wie sie den physiologischen Laboratorien überantwortet werden. Ausserdem mögen hier und da doch wohl Analysen sich eingeschlichen haben, die von Thieren herrühren, an denen schon einmal Versuche gemacht worden sind, während mein hoher Mittelwerth an sicher absolut frischen Thieren erhalten ist.

Obige Tabelle ist eine bündige Widerlegung von Estor und Saint-Pierre. Es versteht sich, dass man immer, wie das diese Forscher ja offenbar stillschweigend auch thun, die Voraussetzung macht, dass die Oxydationsprocesse entweder bei dem Hervortreten des Blutes aus der Ader augenblicklich stille stehen oder dass sich eine gleich grosse Veränderung in beiden Blutarten nach dem Aderlass vollzieht, die sich algebraisch $\mathrm{zu}$ den vorhandenen Werthen addirt, sodass die Differenz constant bleibt. Jene stillschweigende Voraussetzung ist allerdings durch Nichts erwiesen, ja es wäre sehr wohl denkbar, dass, weil leicht verbrennbare Stoffe aus dem Venensystem in das der Arterien übergeführt werden, die Oxydation, nachdem das Blut den Körper verlassen hat, so weit vorschreitet, bis diese Stoffe zerstört sind. Wenn dann im Moment des Aderlasses die Carotis noch mehr solcher leicht oxydirbarer. Stoffe enthielte als die Cruralis, in deren Blut der Oxydationsprocess weiter vorge-

1) E. Pflüger, Die normalen Gasmengen des arteriellen Blutes nach verbesserten Nethoden. Centralblatt f. d. med. Wissenschaften 1867. Nr. 46. 
schritten wäre, so könnte die Carotis wohl factisch reicher an Sauerstoff als die Cruralis sein und die Gasanalyse doch denselben Werth für beide ergeben, da das Blut wegen des immerhin langsamen Entweichens des Sauerstoffs bis zur vollkommenen Entgasung die Zeit behält, diese Stoffe zu verbrennen. Das ist aber ein Punkt, den sich Estor und Saint-Pierre nicht klar gemacht haben. Ich trug dem Rechnung, indem ich den colorimetrischen Versuch mit Carotis und Femoralis anstellte, dem ich deshalb gerade einen besonderen Werth beilegte. Aber obige Versuche beweisen, dass die hypothetische Differenz zwischen dem Blute der A. carotis und femoralis nicht wie Hoppe-Seyler will, durch Sauerstoffabgabe an die Gefässwand bedingt sein kann. Dann müsste sich das Deficit in den Gasanalysen zeigen. Die thatsächlichen Basen der Behauptungen Estor's und Saint-Pierre's sind somit vollkommen in ihrer Werthlosigkeit dargelegt.

Mit Rücksicht auf die Versuche von Hoppe-Seyler, welche dieser Forscher als Stütze der Ansichten von Estor und Saint-Pierre angezogen hat, bleibt uns nun noch Einiges zu bemerken übrig. Es wurde festgestellt, dass frisch aus dem Thiere präparirte Gefässwand bei Körpertemperatur im Laufe einiger Stunden Blut reducirt, mit dem es in Contact ist. Da so lange Zeit nothwendig war, um auch nur die das Gefäss unmittelbar berührende Blutschicht, nicht aber die ganze Masse zu schwärzen, so handelt es sich jedenfalls doch um einen Vorgang, welcher schon seiner Langsamkeit halber nicht zur Erklärung jenes rapiden Sauerstoffverbrauchs angezogen werden kann, der nach den Versuchen von Estor und Saint-Pierre in den Arterien stattfinden soll. Unter den Geweben der Gefässwand wird man aber doch nicht wohl ein anderes denn das Muskelgewebe als wesentlichen Consumenten ansprechen dürfen. Georg Liebig ${ }^{1}$ ), sowie Valentin ${ }^{2}$ ) und Matteucci ${ }^{3}$ ) haben allerdings gefunden, dass aus dem Körper entfernte lebendige, d. h. contractionsfähige Muskeln noch Sauerstoff verbrauchen und Kohlensäure aushauchen. Ludimar

1) G. Liebig, Ueber die Respiration der Muskeln. Müller's Archiv 1850. pag. $393-416$.

2) G. Valentin, Ueber die Wechselwirkung der Muskeln und der sie umgebenden Atmosphäre. Archiv f. physiologische HeilkundeXIV. S. $431-478$.

3) Matteucci, Recherches sur les phénomènes physiques et chimiques de la contraction musculaire. Annal, d. chimie et d. physique XLVII. p. 129-153. 
Hermann ${ }^{1}$ ) zeigte aber, dass, wenn man zwei gleichnamige Muskeln desselben Frosches mit einander auf ihren Gaswechsel vergleicht, von denen der eine während 5-10 Minuten in $45^{\circ}$ warme, der andere in kalte halbprocentige Kochsalzlösung getaucht wird, dann der durch die Wärme starr gewordene beinahe ebenso stark Sauerstoff aufnimmt als der noch zuckungsfähige vorher abgekühlte. Ja der Sauerstoffverbrauch erwies sich sogar grösser bei dem erstarten Muskel, wenn man ihm einfach durch destillirtes Wasser seine Erregbarkeit genommen hatte. Ludimar Hermann ${ }^{2}$ ) sagt deshalb: "Diese Versuche beweisen mit voller Sicherheit, dass der Sauerstoffverbrauch überlebender Muskeln entweder ganz oder doch so weit man messen kann, auf einer Ursache beruht, für die die Lebensprocesse des Muskels keine Rolle spielen. Als diese Ursache lässt sich nichts Anderes als die Oberflächenzehrung angeben. Man hat also aus den Liebig'schen Versuchen mit Muskelathmung einen unberechtigten Schluss gezogen."

Ich habe nun gefunden, dass die aus dem Körper des Thieres entfernte Wand der Arterie saure Reaction darbietet, welche allmählich zuzunehmen scheint, sodass das Blut mit einer von Säuren durchtränkten porösen Masse in Berührung gebracht wird, wenn man den Versuch von Hoppe-Seyler anstellt. Säuren an sich reduciren aber bekanntlich das Blut und es ist durchaus wahrscheinlich, dass wir es hier mit Säuren zu thun haben, die durch die beginnende Todtenstarre des Muskelgewebes der Arterienhaut gebildet sind. Dieser Versuch kann also einem Sauerstoffverbrauch der normalen lebendigen Gefässwand der Arterie innerhalb des normalen Organismus nicht als Beweis dienen.

Somit bliebe uns endlich noch übrig, genauer die auffallenden Angaben Estor's und Saint-Pierre's zu prüfen, um noch bestimmter den Leser darüber aufzuklären, dass bei diesen Forschern auch in den befolgten Methoden und in ihrer Art zu schliessen des Bedenklichen und Fehlerhaften sich genug findet.

In einem und demselben Glasgefässe fangen sie das Blut auf, messen es, treiben den Sauerstoff nach der von Cl. Bernard ange-

1) Ludimar Hermann, Untersuchungen über den Stoffwechsel der Muskeln. ausgehend vom Gaswechsel derselben. Berlin 1867. pag. 32 u. flgd.

2) Ludimar Hermann a. a. 0. pag. 38. 
gebenen Methode mit Kohlenoxydgas aus und bestimmen die Kohlensäure durch Absorption mit Aetzkali, den Sauerstoff mit pyrogallussaurem Kali oder Phosphor ${ }^{1}$ ). Dass die Ausführungen aller dieser Operationen in einer und derselben eigenthümlich Uförmig gebogenen Glasröhre mit Exactheit bei dem Verfahren jener Forscher nicht erreichbar sind, unterliegt keinem Zweifel. Wenn man bei Körpertemperatur längere Zeit arterielles Blut mit einer hinreichenden Menge Kohlenoxydgas heftig schüttelt, dann verbindet sich allerdings alles Blutroth mit diesem Gase und aller Sauerstoff wird aus seiner Verbindung mit dem Haemoglobin in Freiheit gesetzt. $\mathrm{Er}$ entweicht dann zum grössten Theile aus dem Blute, das jetzt seine chemische Anziehung für ihn verloren hat und dessen Absorptionscoefficient zu klein ist, um mehr als eine ganz geringe Menge zurückzuhalten,. welche von der Höhe des Partiardruckes abhängt, den der aus dem Blut in das Kohlenoxydgas entweichende Sauerstoff erreicht. Je grösser die Menge des mit dem Blute in Berührung befindlichen Kohlenoxydgases, um so vollständiger wird der Sauerstoff aus dem Blute entbunden. Estor und Saint-Pierre dürfen nun nicht heftig mit ihrem Apparate das Blut schütteln, weil sonst der entstehende Schaum in die Räume vordringen würde, welche zur Gasanalyse bestimmt sind. Sie Jassen -- und sie meinen dass diess ausreiche - das Blut 7-8 Minuten hin- und herschwanken, während über ihm Kohlenoxydgas in geringer Menge sich vorfindet. Darauf bleibt der Apparat eine halbe Stunde bei mittlerer Temperatur stehen, nach welcher Zeit angenommen wird, dass aller Sanerstoff aus dem Blute ansgetreten sei. Das letztere befindet sich aber, wenn ich die beiden Forscher recht verstehe, in einem schmalen Cylinder, sodass nur die ganz kleine oberflächlichste Schicht der Blutsäule mit Kohlenoxydgas in directe Berührung tritt. Es ist deshalb undenkbar, dass nach so kurzer Zeit und so schwacher Bewegung, die das Schäumen vermeiden soll, der Sauerstoff vollkommen entbunden seí. Hier ist auch die Farbe in keiner Weise ausreichend, um die Sättigung des Blutes mit Kohlenoxyd resp. die

1) Sur un appareil propre aux analyses des mélanges gazenses et spécialement au dosage de gaz du sang, par MM. C. Saint-Pierre et A. Estor, professeurs aggrégés à la Faculté de Montpellier. Journal de l'anatomie et de la physiologie normales et pathologiques de l'homme et des animaux. Paris 1865. pag. 106-108. 
vollkommene Vertreibung des Sauerstoffs festzustellen. Denn das Blut wird nicht schwarz, sondern verändert nur wenig seine Röthe, welche einen blau violetten Ton annimmt, der nicht sehr in die Augen fallend zu sein braucht. Schon Nawrocki (a. a. 0.) hat dargethan, dass nur bei sehr heftigem und lang fortgesetztem Schütteln nahezu der sämmtliche Sauerstoff erhalten werde.

Um zu constatiren, wann die in den Apparat eingeführte, mit pyrogallussaurem Kali getränkte Coakskugel allen Sauerstoff absorbirt hat, richten sich Jene nach der Zeit und nicht wie es allein richtig ist, nach dem Aufhören der Verminderung des Gasvolums bei erneut eingeführter, frisch befeuchteter Absorptionskugel. Wie schon Bunsen hervorhebt, absorbirt zuweilen jenes Salz den Sauerstoff sehr schwierig und stundenlang steht das Niveau des Quecksilbers in dem Absorptionsrohre unbewegt, obwohl noch viel Sauerstoff in dem Gasgemische sich befinden kann. Estor und Saint-Pierre waren deshalb nie sicher, dass aller Sauerstoff thatsüchlich weggenommen sei, wenn sie auch, wie sie sagen, vorher darüber Versuche angestellt hatten, wie schnell ihre Absorptionskugel ein bestimmtes gemessenes Quantum Sauerstoff absorbire. Denn eine solche poröse Kugel nimmt einmal mehr absorbirende Salzlösung als das andere Mal auf, verschluckt vor dem Einführen in den Apparat verschiedene Mengen von atmosphärischem Sauerstoff, was gerade im ersten Momente recht schnell geschicht und zwei scheinbar ganz gleich beschaffene solcher Kugeln können deshalb sehr verschieden geschwind absorbiren. Die Sauerstoffbindung geschieht anfangs sehr schnell, bald aber langsam, sobald die oberflächlichen Flüssigkeitsschichten der Kugel einmal gesättigt sind. Denn jetzt wird der Sauerstoff von der wirksamen Masse durch eine unwirksame die Oberfläche der Kugel überziehende Schicht geschieden.

Neben dem pyrogallussauren Kali gebrauchen Estor und SaintPierre auch den Phosphor zur Absorption des Sauerstoffs, wogegen im Allgemeinen Nichts einzuwenden wäre, wenn sie erstens nicht wieder die Zeit als maassgebend für die Vollendung der Absorption ansehen und wenn sie zweitens vorher nicht dasselbe Rohr, in das die Phosphorkugel eingeführt wird, mit Aetzkalilauge befeuchtet hätten, welche zur Entfernung der Kohlensäure ja immer angewandt zu werden pflegt. In diesem Falle entwickelt sich aber, wovon ich mich direct überzeugte, Phosphorwasserstoff, sodass an Stelle eines verschwindenden Gases ein neues auftritt, welches einen bedeutenden 
Fehler in die Analyse einführt. Hatte der Phosphor vor Erzeugung einer ansehnlichen Menge von Phosphorwasserstoff bereits den grössten Theil des Sauerstoffs absorbirt, so braucht jener auch nicht durch die entstehende Explosion sein Dasein in unangenehmer Weise zu verrathen. Der Phosphor zersetzt bekanntlich für sich allein das Wasser nicht; aber bei Gegenwart von Kali macht sich wegen der Verwandtschaft des letzteren zu der unterphosphorigen Säure eine Zersetzung des Wassers geltend. So entsteht durch Vereinigung von Phosphor mit dem Sauerstoff des Wassers unterphosphorige Säure, die sich mit Kali verbindet, während der aus dem Wasser freiwerdende Wasserstoff einen andern Theil des Phosphors in Beschlag nimmt, um Phosphorwasserstoffgas zu erzeugen. $\mathrm{Zu}$ diesen höchst bedenklichen Punkten kommt dann noch die kleine Blutquantität, welche Estor und Saint-Pierre verwenden und circa $15 \mathrm{CC}$. beträgt, so dass bei der Berechnung der procentigen Menge der begangene Fehler mit einem immerhin sehr grossen Factor multiplicirt wird.

Weitere sehr gewichtige Anhaltspunkte zur Beurtheilung der Schlussfolgerungen von Estor und Saint-Pierre ergeben sich bei genauerer Prüfung der tabellarisch geordneten Zahlenwerthe, welche allen ihren Erörterungen zu Grunde liegen. Ich lasse diese Tabelle deshalb umstehend folgen.

Aus dieser Tabelle berechnen dann Estor und Saint-Pierre, dass die mittleren Variationen des Sauerstoffes in dem Arterienblut die folgenden sind:

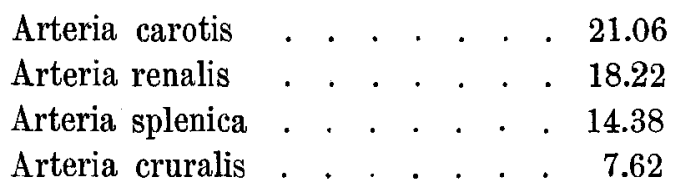

Der eigentliche Hauptfehler, den Estor und Saint-Pierre begehen, liegt darin, dass sie nicht an ein und demselben Thiere das Blut der Arteria carotis und femoralis etc. vergleichen, sondern an verschiedenen Individuen, obwohl es ja doch bekannt ist, wie ausserordentlich der Gehalt an Sauerstoff bei gesunden Thieren schwankt, selbst wenn man immer dieselbe Arterie zum Aderlasse wählt. Statt nun durch eine sehr grosse Zahl von Versuchen diesen Uebelstand zu beseitigen um einen brauchbaren Mittelwerth zu erhalten, machen sie nur wenige Versuche - denn die meisten beziehen sich ja auf Venenblut - ja nehmen einen einzigen Versuch von Bernard über den Sauerstoffgehalt des Carotisblutes ohne Weiteres 
Tabelle von Estor und Saint-Pierre.

Sauerstoffmengen, welche an verschiedenen Punkten des Blutstroms enthalten sind, bezogen auf $100 \mathrm{Vol}$. Blut.

(Diese Werthe der Gasvolumina beziehen sich auf Atmosphärendruck und vermuthlich, worüber Nichts angegeben, auf $0^{\circ}$.)

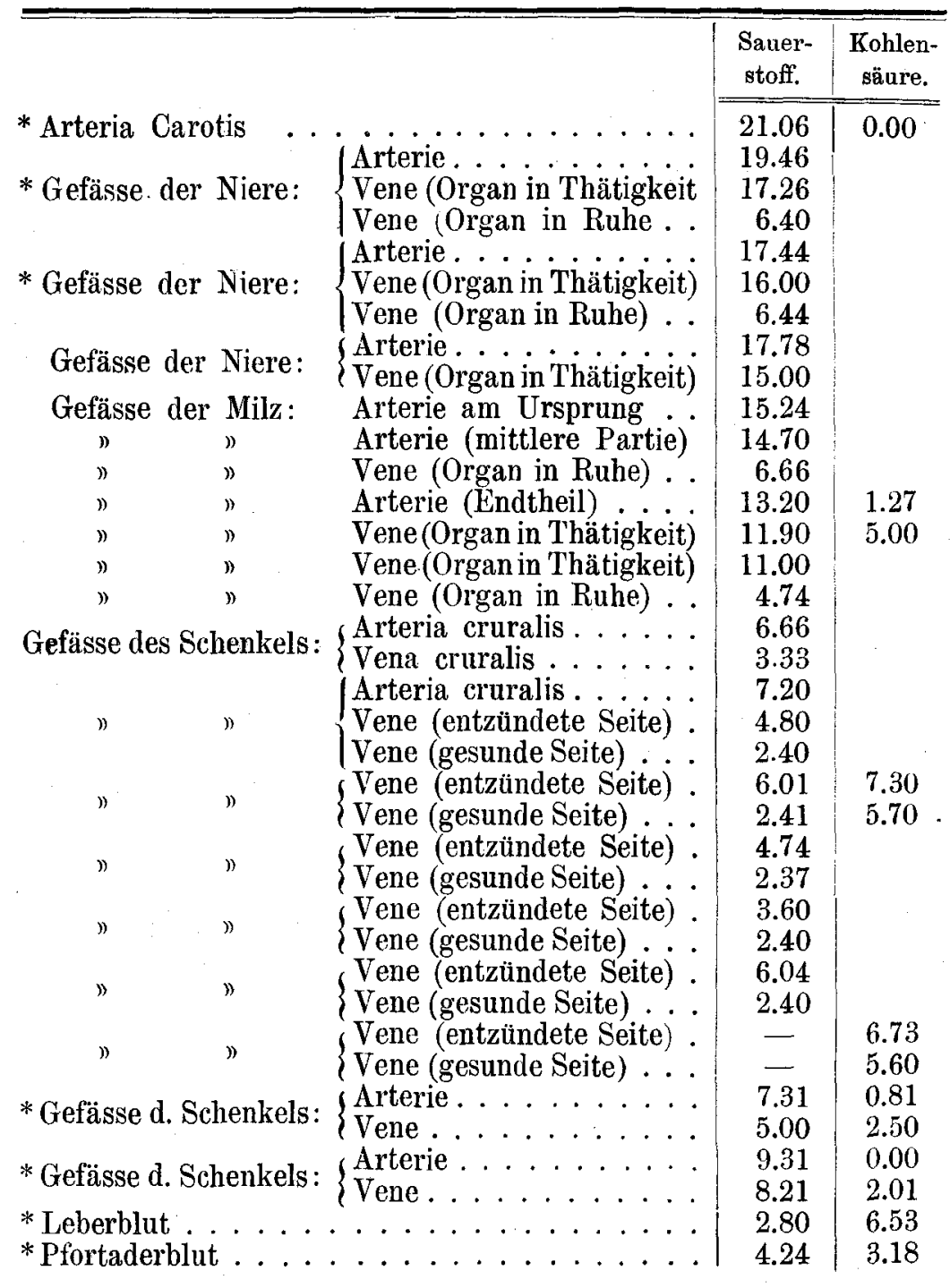

Die mit * markirten Experimente gehören Cl. Bernhard. 
als Mittelzahl. Zufällig ist dieser Werth hoch. Wie Versuche von Ludwig und mir zeigen, kann man ebensogut Hunde erhalten, die nur $10 \%$ Sauerstoff im Blute der Carotis darbieten und solche, in denen in der Arteria femoralis mehr als 21.06 aufgefunden wurde. Der Werth für das Carotisblut hat also keine Bedeutung, weil er ein individueller und kein mittlerer ist. Soviel über den illusorischen Maximalwerth. Die Minimalwerthe beziehen sich auf 4 Analysen vom Blute der Arteria femoralis, von denen zwei von Bernard, zwei von Estor und Saint-Pierre ausgeführt sind. Eine von den 4 Bestimmungen ist deshalb ganz werthlos, weil sie an einem kranken Thiere angestellt ist, bei denen bekanntlich der Sauerstoffgehalt im Allgemeinen niedriger als bei gesunden Thieren gefunden wird. Alle, an denen bereits Operationen gemacht worden sind, ergeben nach meinen Untersuchungen einen verringerten Sauerstoffgehalt. Erst 4-6 Wochen nach der Operation fand ich den früheren Sauerstoffgehalt fast genau wieder. Diese Operationen bestanden in kleinen Aderlässen, nach denen das Gefäss unterbunden wurde. Die Wunde schloss sich durch Eiterung. Verminderung des Sauerstoffs und auch der Kohlensäure sogar während des Fieber's beobachtete ich bei allen diesen Patienten.

Es bleiben also drei Analysen, die möglicherweise ebenfalls mit dem Blute von nicht ganz frischen Hunden angestellt wurden, worüber aber keine Sicherheit vorliegt. Denn weder Estor und Saint-Pierre noch Emile Alglave, der über Bernard's Versuche referirt, geben irgend einen Aufschluss. Da Bernard ${ }^{1}$ ) beabsichtigte, die Veränderung des Gasgehaltes des Blutes, welche durch die Muskelrespiration bedingt ist, $\mathrm{zu}$ demonstriren, so war kein tieferer Grund vorhanden, weshalb er sich nicht eines schon einmal gebrauchten Hundes hätte bedienen dürfen. Wie dem aber auch sei, jedenfalls sind 3 Analysen nicht ausreichend, um bei so grossen Schwankungen des Gasgehaltes eine Mittelzahl zu erhalten. Zufällig konnten gerade diese 3 Hunde schwächliche Thiere sein. Denn es ist ja allbekannt, dass das Blut der Arteria femoralis 2-3mal so viel Sauerstoff enthalten kann, als in diesen Versuchen gefunden wird. Einer der oben von mir mitgetheilten zeigt einen Gehalt von $18.7 \%$, d. h. nach

1) Physiologie générale. Cours de M. Claude Bernard etc. Respiration musculaire. - Suc musculaire. Rigidité cadavérique. Revue des cours scientifiques de la Franee et de l'étranger. 24 September 1864. pag. 621-624. 
Umrechnung auf den von Estor und Saint-Pierre zu Grunde gelegten Druck, von $24.6 \%$, also bei Weitem mehr als von jenen Forschern für die Carotis angegeben wird. Ich habe noch öfter Sauerstoffwerthe, die diesem für arterielles Blut überhaupt ausserordentlich hohen nahe kommen, im Blute constatirt, welches der Arteria femoralis entnommen war. Es ist gewiss und immerhin wichtig, dass die Maximalwerthe, welche das Blut der letzteren Arterie liefert, nicht geringer sind als die für die Carotis festgestellten. Somit ist es rein zufällig, dass bei den drei von Estor und Saint-Pierre gebrauchten Thieren das Blut der Femoralis einen so niedrigen Werth ergab; bei denselben Thieren würde das Carotisblut keinen höheren geliefert haben.

Ich kann nicht umhin, hier auf einen Umstand aufmerksam zu machen, welcher unter Umständen dahin wirkt, dass das aus der Carotis erhaltene Blut etwas reicher an Sauerstoff und ärmer an Kohlensäure gefunden werden kann, als das der Femoralis entnommene. Die Femoralis liegt viel oberflächlicher und lässt sich durch schnellere und kürzere Operation erreichen, als das meist bei der A. Carotis der Fall ist. Auch reagiren die Hunde stärker auf Verwundungen am Halse. Sie haben deshalb gewöhnlich bei Blosslegung der Carotis viel mehr getobt als bei der der Femoralis. Es ist aber gewiss, dass heftige Athmung den Sauerstoffgehalt des Blutes etwas steigert, die Kohlensäure vermindert. Auch aus diesem Grunde muss man" gleichzeitig an demselben Thiere beide Arterien anzapfen.

Bei der ferneren Untersuchung des Blutes der Arteria renalis und splenica ergeben sich nun die gewöhnlichen mittleren Werthe für den Sauerstoffgehalt, welcher also, da zufällig die Zahl für die Femoralis abnorm niedrig war, höher als dieser letztere ausfällt, sodass das Gesetz für Estor und Saint-Pierre zu stimmen schien. Es ergiebt sich nur etwa der Widerspruch, dass schon an der Wurzel der Arteria splenica, die doch dem Herzen näher als die Arteria renalis ist, ein geringerer Sauerstoffgehalt, als in dieser gefunden wird. Die eigentliche Blüthe der ganzen Arbeit gipfelt aber noch in dem Nachweise, dass der Sauerstoffgehalt am Ursprung der Arteria splenica $15.24 \%$, in der mittleren Partie $14.7 \%$ und in der Endpartie $13.2 \%$ betrage. Diess ist allerdings nur eine Serie, bei der nicht angegeben wurde, $o b$ von demselben oder verschiedenen Thieren. Nicht ein zweites Mal wird es je wieder einem Beobachter gelingen, durch den Zufall diese Zahlen sich so gruppiren zu sehen. Es ist somit bewiesen, dass die Resultate, welche Estor und 
Saint-Pierre erhalten haben, mit unzuverlässigen Methoden gewonnen und darum durchaus nicht beweiskräftig erscheinen. Aber gesetzt auch, dass diese an verschiedenen Thieren ermittelten Werthe als sicher zugegeben würden, beweisen sie nicht was sie sollen, weil einmal aus nur einem, ein andermal aus nur drei Versuchen gerade die wichtigsten Mittelwerthe gezogen sind, welche bei den ungeheuren Schwankungen des Gasgehaltes natürlich sehr weit vom wahren Mittel entfernt sein können, sodass eine Vergleichung solcher falschen Mittelwerthe dann zu den irrigsten Folgerungen benutzt werden konnte.

\section{Uober anomale Farbenempfindungen und die phy- siologischen Grundfarben.}

Von

W. Preyer.

Es sollen in dieser Abhandlung mitgetheilt werden zunächst Beobachtungen über den Einfluss des gelben Farbstoffs an der Stelle des deutlichsten Sehens auf das Zustandekommen von Farbenempfindungen, sodann einige Untersuchungen über Farbenblindheit, insbesondere Grünblindheit. Hieran schliesst sich ein Versuch die drei Grundfarben etwas genauer, als es bisher geschehen ist, zu ermitteln.

1. Ueber den Einfluss des gelben Pigmentes der macula lutea auf Farbensehen.

Nur wenige bestimmte Angaben liegen über diese Frage vor. Maxwell ${ }^{1}$ ) fand seine Augen für den zwischen den Linien $\mathrm{E}$ und $\mathrm{F}$

1) On the theory of compound colours. Phil. Trans, for the year 1860. London 1861 p. $76,77$. 